\title{
Pathogenesis of prostatic small cell carcinoma involves the inactivation of the P53 pathway
}

\author{
Hongbing Chen ${ }^{1,2 *}$, Yin Sun ${ }^{2 *}$, Chengyu $\mathrm{Wu}^{5}$, Clara E Magyar ${ }^{2}$, Xinmin $_{\mathrm{Li}} \dot{i}^{2,3}$, \\ Liang Cheng ${ }^{6}$, Jorge $L \mathrm{Yao}^{7}$, Steven Shen ${ }^{8}$, Adeboye O Osunkoya ${ }^{9}$, \\ Chaozhao Liang ${ }^{1}$ and Jiaoti Huang ${ }^{2,3,4}$
}

\footnotetext{
${ }^{1}$ Department of Urology, The Geriatrics Research Institute, First Affiliated Hospital of Anhui Medical University, Anhui, China ${ }^{2}$ Department of Pathology and Laboratory Medicine, ${ }^{3}$ Jonsson Comprehensive Cancer Center and ${ }^{4}$ Broad Center for Regenerative Medicine and Stem Cell Biology, David Geffen School of Medicine at UCLA, 10833 Le Conte Avenue, 13-229 CHS, Los Angeles, California 90095-1732, USA

${ }^{5}$ Transplant Immunology Laboratory, Albert Einstein College of Medicine, Montefiore Medical Center, Bronx, New York, USA

${ }^{6}$ Department of Pathology, Indiana University School of Medicine, Indianapolis, Indiana, USA

${ }^{7}$ Department of Pathology, University of Rochester School of Medicine, Rochester, New York, USA

${ }^{8}$ Department of Pathology, The Methodist Hospital, Houston, Texas, USA

${ }^{9}$ Department of Pathology, Emory University School of Medicine, Atlanta, Georgia, USA

(Correspondence should be addressed to J Huang at Department of Pathology and Laboratory Medicine, David Geffen School of Medicine at UCLA; Email: jiaotihuang@mednet.ucla.edu; C Liang; Email: liang_chaozhao@163.com)

${ }^{*}(\mathrm{H}$ Chen and $\mathrm{Y}$ Sun contributed equally to this work)
}

\begin{abstract}
Small cell neuroendocrine carcinoma (SCNC) of the prostate is a variant form of prostate cancer that occurs de novo or as a recurrent tumor in patients who received hormonal therapy for prostatic adenocarcinoma. It is composed of pure neuroendocrine (NE) tumor cells, but unlike the scattered NE cells in benign prostate and adenocarcinoma that are quiescent, the NE cells in SCNC are highly proliferative and aggressive, causing death in months. In this study, we provide evidence that interleukin 8 (IL8)-CXCR2-P53 (TP53) signaling pathway keeps the NE cells of benign prostate and adenocarcinoma in a quiescent state normally. While P53 appears to be wildtype in the NE cells of benign prostate and adenocarcinoma, immunohistochemical studies show that the majority of the NE tumor cells in SCNC are positive for nuclear p53, suggesting that the $p 53$ is mutated. This observation is confirmed by sequencing of genomic DNA showing p53 mutation in five of seven cases of SCNC. Our results support the hypothesis that $p 53$ mutation leads to inactivation of the IL8-CXCR2-p53 signaling pathway, resulting in the loss of an important growth inhibitory mechanism and the hyper-proliferation of NE cells in SCNC. Therefore, we have identified potential cells of origin and a molecular target for prostatic SCNC that are very different from those of conventional adenocarcinoma, which explains SCNC's distinct biology and the clinical observation that it does not respond to hormonal therapy targeting androgen receptor signaling, which produces short-term therapeutic effects in nearly all patients with prostatic adenocarcinoma.
\end{abstract}

Endocrine-Related Cancer (2012) 19 321-331

\section{Introduction}

Prostate cancer (PC) is the most common malignancy in men and the second leading cause of cancer-related deaths (Cooperberg et al. 2004). Normal prostate epithelium contains luminal epithelial cells, basal cells, and a minor component of neuroendocrine (NE) cells that are scattered throughout the prostate (Vashchenko \& Abrahamsson 2005, Huang et al. 2007, Yuan et al. 2007, Sun et al. 2009). The majority of PCs are classified as adenocarcinomas characterized by absence of basal cells and uncontrolled proliferation of malignant tumor cells with luminal cell features 
including glandular formation and expression of androgen receptor (AR) and prostate-specific antigen (PSA). Interestingly, every case of prostatic adenocarcinoma also contains a small population (usually $\sim 1 \%$ ) of NE cells (Vashchenko \& Abrahamsson 2005, Huang et al. 2007, Yuan et al. 2007, Sun et al. 2009). The NE cells in adenocarcinoma share many important features with those in the benign prostate. In contrast to the luminal-type non-NE tumor cells, the NE tumor cells do not express AR and PSA (Bonkhoff 2001, Huang et al. 2006). Importantly, unlike the bulk, non$\mathrm{NE}$ tumor cells, NE cells in benign prostate and adenocarcinoma are normally quiescent (Bonkhoff 2001, Huang et al. 2006).

While adenocarcinomas comprise the majority of $\mathrm{PC}$, there are variant forms among which small cell neuroendocrine carcinoma (SCNC) is an important histological subtype that is often seen in patients with advanced disease. Prostatic SCNC is composed of tumor cells with NE phenotype (Grignon 2004). In comparison to adenocarcinoma, which usually shows glandular formation, SCNC has a solid, sheet-like growth pattern but no glandular formation. Tumor cells are small with fine chromatin pattern, scant cytoplasm, and nuclear molding. Mitotic figures, crush artifact, and tumor necrosis are frequent findings (Yao et al. 2006, Huang et al. 2007, Wang \& Epstein 2008). Although SCNCs may arise de novo, such tumors are often seen as recurrent tumors after hormonal therapy for conventional adenocarcinomas of the prostate (Miyoshi et al. 2001, Tanaka et al. 2001). The prevalence of this disease is likely underestimated because patients with advanced and metastatic diseases usually do not undergo tissue diagnosis. Accurate diagnosis of SCNC is important as such tumors do not respond to hormonal therapy targeting the AR signaling pathway (Brown et al. 2003), which produces a short-term therapeutic effect in nearly all cases of prostatic adenocarcinoma (Scher 2003).

The NE tumor cells in prostatic SCNCs share many features with those in benign prostate and prostatic adenocarcinoma. For example, they contain dense-core secretory granules when examined by electron microscopy and are usually positive for NE markers chromogranin A ( $\mathrm{CgA})$ and synaptophysin by immunohistochemistry. However, unlike the NE cells in benign prostate and prostatic adenocarcinoma that are quiescent, the NE tumor cells in prostatic SCNCs are highly proliferative, leading to early metastasis (Erasmus et al. 2002), and the patients usually die within months of diagnosis. The molecular mechanisms responsible for the aggressive biological behavior of the NE tumor cells in prostatic SCNC remain unknown. Here, we provide evidence that the interleukin 8 (IL8)-CXCR2-p53 (TP53) pathway may be important in keeping the NE cells in benign prostate and prostatic adenocarcinoma in a quiescent state, and inactivation of the pathway due to $p 53$ mutation may be responsible for the rapid proliferation of $\mathrm{NE}$ cells in prostatic SCNC.

\section{Materials and methods}

\section{Cell culture and transfection}

LNCaP cells were maintained in RPMI-1640 medium supplemented with $10 \%$ fetal bovine serum, $2 \mathrm{mM}$ L-glutamine, penicillin $(100 \mathrm{U} / \mathrm{ml})$, and streptomycin $(100 \mu \mathrm{g} / \mathrm{ml})$ in a humidified atmosphere of $5 \% \mathrm{CO}_{2}$ maintained at $37^{\circ} \mathrm{C}$. pcDNA3 vector or pcDNA3CXCR2 was transfected into LNCAP cells with Lipofectamine 2000 (Invitrogen), and stable clones were selected with $\mathrm{G} 418$ at $300 \mu \mathrm{g} / \mathrm{ml}$ for 30 days. For siRNA transfection, Dharmafect \#2 was used with siRNA at $100 \mathrm{nM}$ for $p 53$ (duplex sequences are 5'-rCrCrA rCrCrArUrCrCrArCrUrArCrArArCrUrArCrArUrGTG-3', 5'-rCrArCrArUrGrUrArGrUrUrGrUrArGrUrGrGrArUrGrGrUrGrGrUrA- $3^{\prime}$ ). For expression of p53 in PC3 cells, GFP expression plasmid with a ratio of 9:1 to CMV-driven $p 53$ plasmid were transfected with Fugene 6, 3 days later, IL8 was added, and the number of GFP-positive cells were counted using Acumen laser cytometer.

\section{Immunoblot analysis}

Cells were washed with PBS and lysed in RIPA buffer (50 mM Tris, $\mathrm{pH} 7.4,150 \mathrm{mM} \mathrm{NaCl}, 1 \%$ Triton X-100, $0.5 \%$ deoxycholate, $0.1 \%$ SDS) containing protease inhibitor cocktail for $15 \mathrm{~min}$ at $4{ }^{\circ} \mathrm{C}$. Cell lysates were centrifuged and supernatants were collected. Equal amounts of proteins were resolved on SDS-PAGE gels and transferred to polyvinylidene fluoride (PVDF) membranes. The resulting blots were blocked in 5\% nonfat dry milk for $30 \mathrm{~min}$ followed by incubation with primary antibody against CXCR2 (Cat. no. 555932; BD Biosciences, San Diego, CA, USA). HRPconjugated secondary antibody and supersignal west pico chemiluminescent substrate (Thermo Fisher Scientific, Waltham, MA, USA) were used to visualize antigen-antibody complexes.

\section{Flow cytometry analysis}

Flow cytometry analysis was performed as described previously (Palapattu et al. 2009) using a fluorescein 
isothiocyanate (FITC) conjugated anti-CXCR2 antibody (BD Biosciences).

\section{Cell proliferation assay}

Cells were seeded into 96-well microplates and $10 \mathrm{nM}$ IL8 were added to the culture $24 \mathrm{~h}$ after seeding for 6 days with the cell number determination at days 0,2 , 4, and 6 using CellTiter Glo assay (Promega) according to the manufacture's protocol.

\section{RNA isolation and quality control and microarray hybridization}

Total RNA was isolated using the RNeasy Mini Kit (Qiagen) according to the manufacturer's instructions. RNA integrity was evaluated using an Agilent 2100 Bioanalyzer (Agilent Technologies, Palo Alto, CA, USA) and purity/concentration was determined using a NanoDrop 8000 (NanoDrop Products, Wilmington, DE, USA). Microarray targets were prepared using MessageAmp Premier RNA Amplification Kit (Ambion) and hybridized to the Affymetrix GeneChip U133plus 2.0 Array (Affymetrix, Inc., Santa Clara, CA, USA) according to the manufacturers' instructions. The arrays were washed and stained with streptavidin phycoerythrin in Affymetrix Fluidics Station 450 using the Affymetrix GeneChip protocol and then scanned using an Affymetrix GeneChip Scanner 3000.

\section{Microarray data analysis}

The acquisition and initial quantification of array images were conducted using the AGCC Software (Affymetrix, Inc.). The subsequent data analyses were performed using Partek Genomics Suite version 6.4 (Partek, Inc., St. Louis, MO, USA). Differentially expressed genes were selected at $\geq 1.5$-fold and $P<0.05$. Cluster analyses and principal component analysis were conducted with Partek default settings. Biofunctional analysis was performed using Ingenuity Pathways Analysis Software version 7.6 (Ingenuity Systems, Redwood City, CA, USA).

\section{Construction of tissue microarrays and immunohistochemical staining}

Construction of tissue microarray (TMA) and immunohistochemical staining has been described previously (Huang et al. 2005). Three hundred cases of prostatectomy specimens were reviewed and representative cancer and benign areas were circled. The cancer and benign prostate tissues were then transferred from donor blocks to recipient blocks to construct tissue microarray blocks. H\&E sections were prepared from the top for quality control.
For immunohistochemical studies, paraffin sections of $5 \mu \mathrm{m}$ thickness were prepared from regular histological blocks or from tissue microarrays. The sections were deparaffinized with xylene and rehydrated through graded ethanol. Endogenous peroxidase activity was blocked with $3 \%$ hydrogen peroxide in methanol for $10 \mathrm{~min}$. Heat-induced antigen retrieval (HIER) was carried out for all sections in $0.01 \mathrm{M}$ citrate, $\mathrm{pH}=6.00$, using a vegetable steamer at $95{ }^{\circ} \mathrm{C}$ for $25 \mathrm{~min}$. Mouse monoclonal anti-p53 antibody (clone 1801, Oncogene, OP09-100 $\mu \mathrm{g}$, used at 1:50) and mouse monoclonal anti-CgA antibody (clone DAK-A3, M0869, used at 1:1000; DakoCytomation Carpinteria, CA, USA) were used as primary antibodies. MACH2 Mouse HRPPolymer (Cat no. \#MHRP520L, Biocare Medical, Concord, CA, USA) was used as the secondary antibody. For double staining of p53 and $\mathrm{CgA}$, the two antibodies were used sequentially. 3,3'-diaminobenzidine (DAB, brown) and alkaline phosphatase (AP, red) were used as chromogens.

\section{Genomic sequencing of p53 exons}

Based on microscopic examination, small cell carcinoma regions in paraffin section were scraped off and collected from the slides followed by deparaffinization and overnight proteinase $\mathrm{K}$ digestion. The genomic DNA was purified through ethanol precipitation after phenol and chloroform extraction. Exons 5-10 of p53 were amplified by PCR followed by gel electrophoresis analysis. The PCR products were either directly used for sequencing if there is specific amplification or gel purified and sequenced by one of the amplifying primers. Primers for amplifications are as follows: exon 5 forward $5^{\prime}$-GCCCTGTCGTCTCTCCAG-3', reverse $5^{\prime}$-GACTTTCAACTCTGTCTCCTTCC-3'; exon 6 forward $5^{\prime}$-CTTAACCCCTCCTCCCAGAG-3', reverse $5^{\prime}$-CAGGCCTCTGATTCCTCACT-3'; exon 7 forward 5'-GTGTGCAGGGTGGCAAGT-3', reverse $5^{\prime}$-CGACAGAGCGAGATTCCATC- $3^{\prime}$; exons 8 and 9 forward 5'-CGGCATTTTGAGTGTTAGACTG-3', reverse $5^{\prime}$-GCCTCTTGCTTCTCTTTTCC-3', exon 10 forward $5^{\prime}$-TGCATGTTGCTTTTGTACCG-3', reverse 5'-GGAGTAGGGCCAGGAAGG-3'.

\section{Results}

\section{Establishing LNCaP cell lines stably expressing IL8 receptor CXCR2}

We have previously shown that NE cells in benign prostate and prostatic adenocarcinoma express IL8 and its receptor CXCR2 (Huang et al. 2005). We hypothesized that the autocrine action of IL8-CXCR2 
may be responsible for certain function of NE cells (Huang et al. 2005), but the exact consequence of this autocrine action was unclear. A more recent publication showed that activation of CXCR2 by IL8 leads to cellular senescence, a state of stable proliferative arrest, via a p53-dependent mechanism (Acosta et al. 2008). Thus, we further hypothesized that IL8-CXCR2-p53 pathway may also be active in NE cells in benign prostate and prostatic adenocarcinoma, which maintains these cells in a quiescent state.

In human PC tissue, NE cells are positive for IL8 and its receptor CXCR2 while luminal cells are negative for both (Huang et al. 2005). LNCaP cells have features of prostatic cancer cells with luminal (non$\mathrm{NE}$ ) features in that they express luminal differentiation markers AR and PSA but not NE markers CgA and neuron-specific enolase (NSE) (Palapattu et al. 2009). Accordingly, there is no detectable expression of IL8 and a very low level of CXCR2 in LNCaP cells.

In order to study the function and signaling pathway of CXCR2, we transfected LNCaP cells with pcDNA3CXCR2 plasmid to obtain LNCaP cells that stably overexpress CXCR2. Figure 1 (A, B and C) shows that we have established $\mathrm{LNCaP}$ cells that overexpress CXCR2 (LNCaP/CXCR2 cells), as confirmed by immunoblot as well as flow cytometric analysis with antibodies against CXCR2.

\section{Activation of CXCR2 by IL8 inhibits cell proliferation}

In a genetic screen to identify molecules that are critical for replicative senescence, it was found that activation of CXCR2 by IL8 leads to cellular senescence, which is mediated by p53 (Acosta et al. 2008). Thus, we hypothesized that in NE cells of benign prostate and adenocarcinoma, the IL8CXCR2-p53 pathway may be responsible for keeping the cells in a quiescent state normally. To test this hypothesis, we used LNCaP and LNCaP/CXCR2 cells and examined their proliferative responses to IL8. As shown in Fig. 1D, IL8 inhibited the proliferation of LNCaP/CXCR 2 cells but not the parental LNCaP cells, a finding that supports our hypothesis. We also examined the transcriptional responses to IL8 by comparing the transcriptional profiles of cells with or without IL8 treatment. As shown in Fig. 1E, IL8 stimulation induced similar changes in transcriptional profiles at 1 and $12 \mathrm{~h}$, with up- or downregulation of a variety of genes. Interestingly, activation of CXCR2 by IL8 led to altered expression of many genes involved in p53 signaling. Functional gene family analysis showed that among the genes whose expression increased significantly in response to IL8 stimulation were those controlling the G1/S and G2/M transition check points. As an important function of p53 is in the cell cycle control and growth inhibition, these results suggest that growth inhibition of LNCaP/CXCR2 cells after activation of CXCR2 by IL8 may involve the p53 pathway.

\section{P53 is required for the growth inhibitory function of the IL8-CXCR2 autocrine pathway}

P53 was shown to be critical in mediating the IL8CXCR2 signaling to induce cellular senescence (Acosta et al. 2008). Similarly, p53 may also be essential in the growth suppression after activation of CXCR2 by IL 8 treatment in LNCaP/CXCR 2 cells, as suggested by our microarray studies. To directly test this hypothesis, we attempted to abrogate $\mathrm{p} 53$ function in LNCaP/CXCR2 cells to determine whether a loss of $\mathrm{P} 53$ activity would change the growth response toward IL8 treatment. P53 protein is of wild-type in LNCaP cells (van Bokhoven et al. 2003), thus inactivation of p53 activity can be achieved through reduction of the endogenous wild-type protein. We used a transient transfection method to introduce siRNA to reduce the endogenous P53 level in LNCaP/CXCR2 cells. As shown in Fig. 2A, we were able to significantly reduce the P53 level by siRNA method. Importantly, IL8 treatment of the cells with reduced P53 levels resulted in an increase in cell number while the control cells showed reduced cell numbers in response to IL8 treatment, suggesting that P53 is critically important for the growth inhibition after activation of CXCR2 by IL8. The results also suggest that in the absence of functional P53, activation of CXCR2 by IL8 may stimulate cell proliferation via a different signaling pathway.

Our group has recently demonstrated that another commonly used PC cell line, PC3, possesses features of prostatic SCNC in that these cells are negative for luminal differentiation markers AR and PSA but positive for NE markers (Tai et al. 2011). Similar to NE cells in benign prostate and prostatic adenocarcinoma, PC3 cells express IL8 (Ma et al. 2009) and CXCR2 (Reiland et al. 1999). Contrary to NE cells in benign prostate and prostatic adenocarcinoma, PC3 cells are highly proliferative NE cells similar to human prostatic SCNC. We hypothesized that loss of the growth inhibitory function of the IL8-CXCR2-p53 pathway may be responsible for the rapid proliferation and aggressive behavior of PC3 cells. In support of our hypothesis, it has been reported that PC3 cells, unlike LNCaP cells, contain a p53 mutation 
A

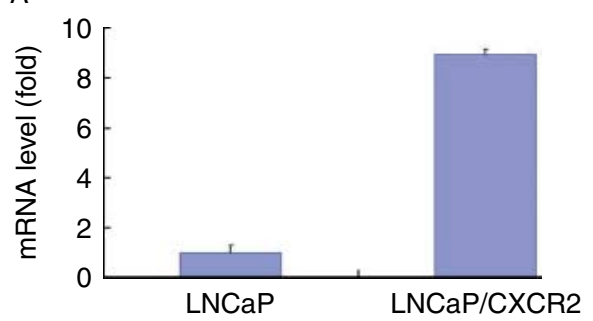

B

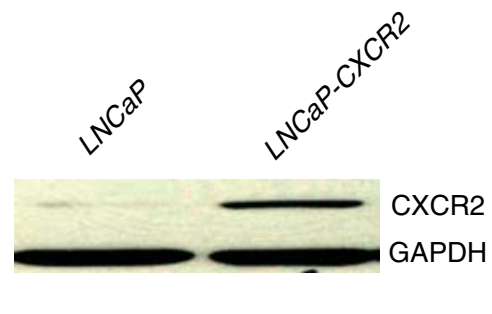

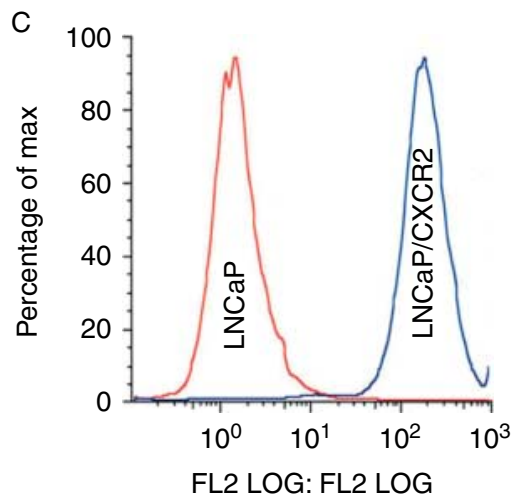

D

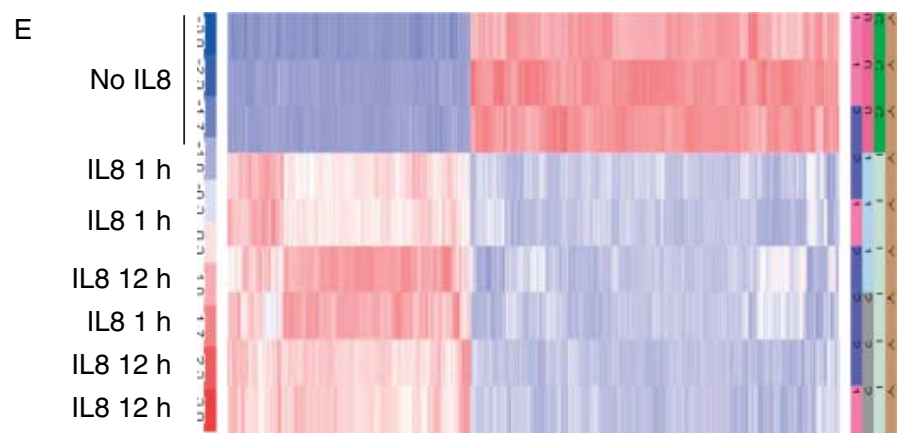

Figure 1 Activation of CXCR2 by IL8 inhibits cell proliferation and induces changes in gene expression profile. LNCaP cells were stably transfected with a plasmid-expressing CXCR2, a receptor for IL8. Real-time PCR (A), western blot (B), and flow cytometry (C) analyses show that CXCR2 is not expressed in the parental LNCaP cells but highly overexpressed in the stable cell line. (D) LNCaP cells transfected with vector control or CXCR2 were cultured in the absence or presence of IL8. IL8 significantly inhibits the proliferation of LNCaP cells expressing CXCR2. (E) Microarray analysis shows that IL8 stimulation of LNCaP/CXCR2 cells results in significant changes of gene expression profile. Similar changes were observed at 1 and $12 \mathrm{~h}$ after IL8 treatment.

(van Bokhoven et al. 2003). Therefore, we tested whether forced expression of wild-type P53 may restore the function of the IL8-CXCR2-P53 pathway resulting in inhibition of cellular proliferation. Similar to what was reported previously (Isaacs et al. 1991), introduction of wild-type P53 significantly slowed the growth of PC 3 cells. Additionally, in contrast to control PC 3 cells, IL8 treatment of PC3 cells expressing wild-type P53 led to reduced cell numbers. These data suggest that IL8-CXCR2-p53 pathway plays a central role in mediating growth suppression normally, and loss of function of this pathway (such as a P53 mutation) can lead to increased proliferation in NE cells.

\section{Mutation of $P 53$ in NE tumor cells of prostatic SCNC}

NE cells in benign human prostate and prostatic adenocarcinoma are quiescent (Huang et al. 2007) but those in prostatic SCNC, whether arising de novo or as a recurrent tumor in patients treated with hormonal therapy for adenocarcinoma, are highly proliferative and biologically very aggressive (Yao et al. 2006, Wang \& Epstein 2008). The underlying molecular mechanism responsible for such a difference is unclear. The results presented above are consistent with the model that the intact IL8-CXCR2-P53 pathway may be responsible for keeping the NE cells 


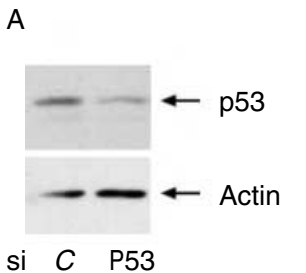

si $\quad C \quad$ P53

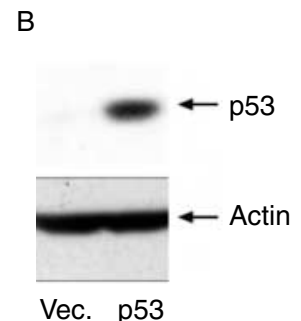

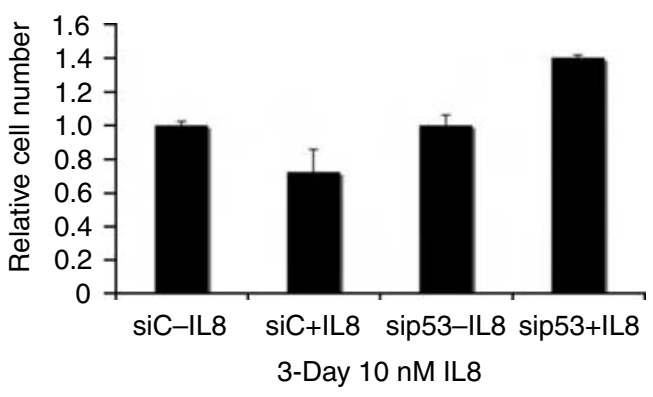

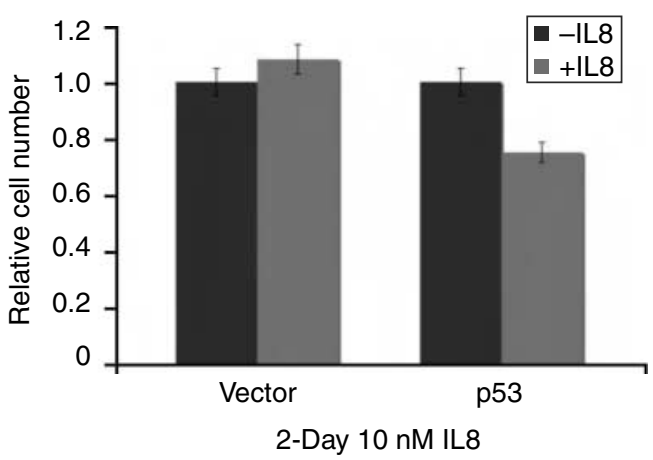

Figure 2 p53 is important for mediating IL8 activity in LNCAP and PC3 cells. (A) Knocking down endogenous p53 in CXCR2expressing LNCaP cells leads to increase in cell proliferation in response to IL8 treatment. (B) Expression of p53 renders PC3 cells responsive to growth inhibition of IL8. GFP-positive cells were counted using laser microplate cytometer as equivalent to p53 expression in PC3 cells. The ratio of the number of cells in the presence or absence of IL8 was tabulated.

under a state of quiescence, a mechanism that has been reported in senescent cells (Acosta et al. 2008). Therefore, we further hypothesized that the reason the NE tumor cells in prostatic SCNC are highly proliferative and biologically aggressive is because this important growth-inhibitory pathway is inactivated in such cells. As mutation of P53 represents the most common genetic alteration of human malignancies, we further hypothesized that P53 mutation is a fundamental molecular change responsible for the aggressive biological behavior of prostatic SCNC as has been observed in PC3 cells, a cell line that is characteristic of human prostatic SCNC (Tai et al. 2011).

It is well known that $p 53$ mutation causes prolonged half-life and subsequent nuclear accumulation of the p53 protein, which then becomes detectable by immunohistochemistry (Schlomm et al. 2008, Kudahetti et al. 2009). Therefore, we performed immunohistochemistry to observe p53 nuclear staining in NE cells of benign prostate, prostatic adenocarcinoma, and SCNC. Adjacent sections were prepared from tissue microarrays containing 150 cases (300 cores) of prostatic adenocarcinoma and an equal number of benign prostate from the same patients. We had shown that the two adjacent sections prepared in this manner contained essentially identical cells and such sections are useful in identifying proteins specifically expressed in prostatic NE cells (Huang et al. 2005, 2006, Wu et al. 2006). To determine whether NE cells in benign prostate tissue and adenocarcinoma are positive for nuclear p53 staining, the first sections from the tissue microarrays were stained with an anti-CgA antibody to highlight NE cells as $\mathrm{CgA}$ is widely considered the most sensitive and specific marker for prostatic NE cells (Vashchenko \& Abrahamsson 2005, Huang et al. 2007, Yuan et al. 2007) and the second sections stained with an anti-p53 antibody. The stained slides were scanned and analyzed using the Ariol SL-50 scanner with thresholds applied for RGB algorithms, shapes, and sizes. The number of nuclei and positively stained cells were counted and separately recorded. Benign cores $(n=300)$ in the TMAs contained 1353 NE cells (representing $0.33 \%$ of total cells) and 3297 p53+ cells (representing $0.84 \%$ of total nuclei); cancer cores $(n=300)$ had $1331 \mathrm{NE}$ cells (representing $0.23 \%$ of total cells) and $4646 \mathrm{p} 53+$ cells (representing 0.9\% of total nuclei). As has been described previously, the NE cells were present as individual cells or small clusters (Huang et al. 2007). The p53-positive cells in benign prostate appear to be mostly basal cells while in adenocarcinoma, the p53-positive cells were 
mostly individual tumor cells. Importantly, when the corresponding cores in the two TMA sections were matched and compared, there was no overlap between $\mathrm{CgA}+$ and $\mathrm{p} 53+$ cells, supporting the conclusion that the NE cells were negative for $\mathrm{p} 53$ nuclear staining and the scattered nuclear $\mathrm{p} 53+$ cells were not the NE cells (Fig. 3A and B). To confirm this finding, we also performed double staining for $\mathrm{CgA}$ and $\mathrm{p} 53$ on the same TMA section. We again showed that $\mathrm{CgA}$ staining (cytoplasmic) and p53 staining (nuclear) did not exist in the same cells (Fig. 3C). These results suggest that in benign prostate and prostatic adenocarcinoma, the NE cells likely have wild-type p53.

To determine whether $p 53$ mutation may be present in prostatic SCNC, we used immunohistochemistry to study p53 expression in 31 cases of prostatic SCNC using regular histological sections. Unlike NE cells in benign prostate or prostatic adenocarcinoma, the NE tumor cells
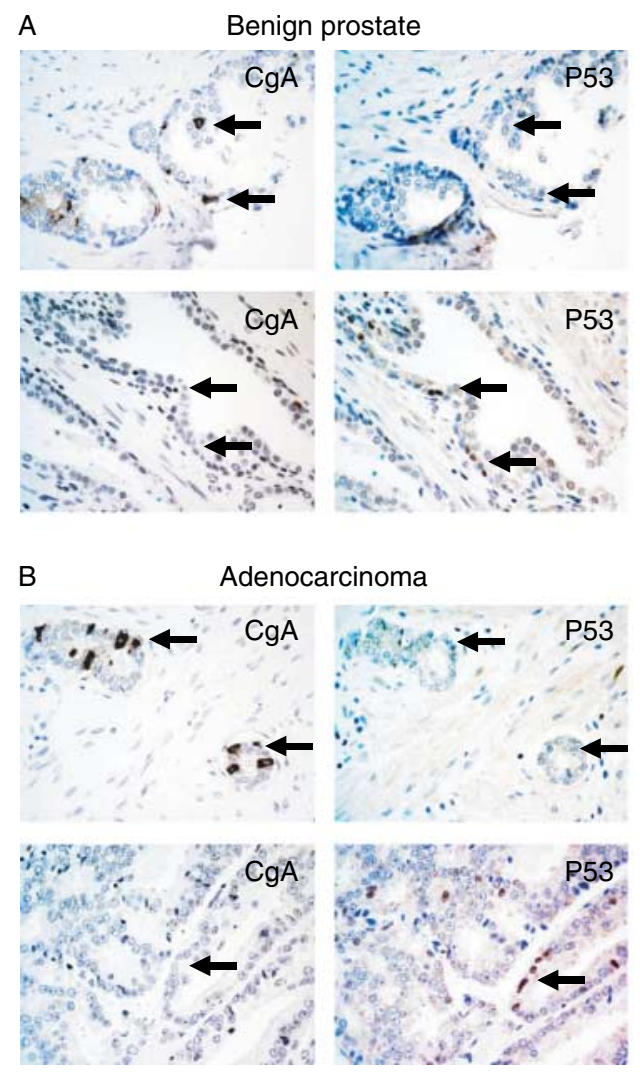

in prostatic SCNCs were positive for $\mathrm{p} 53$, with $74 \%$ (23/31) of the cases showing positivity in $50-100 \%$ cells and the remaining $26 \%$ (8/31) of the cases with positivity in 5-50\% cells (Fig. 4A and B). As nuclear staining of p53 results from prolonged half-life of the protein after mutations, these results suggest that most, if not all, prostatic SCNCs contain mutations in the $p 53$ gene.

To directly examine the possible mutations of the p53 gene in the SCNC, we obtained formalin-fixed, paraffin-embedded tissue from seven cases of prostatic SCNC and extracted genomic DNA from them. Exons 5-10 of $p 53$ were amplified by PCR followed by direct sequencing. As shown in Fig. 4C, this analysis indicated that five out of seven tumor samples contain an identical allele of a missense transition converting $\mathrm{G}$ to $\mathrm{A}$ at position 747 , changing negatively charged aspartic acid to hydrophilic amino acid asparagine at amino acid 184, again supporting our hypothesis that

C
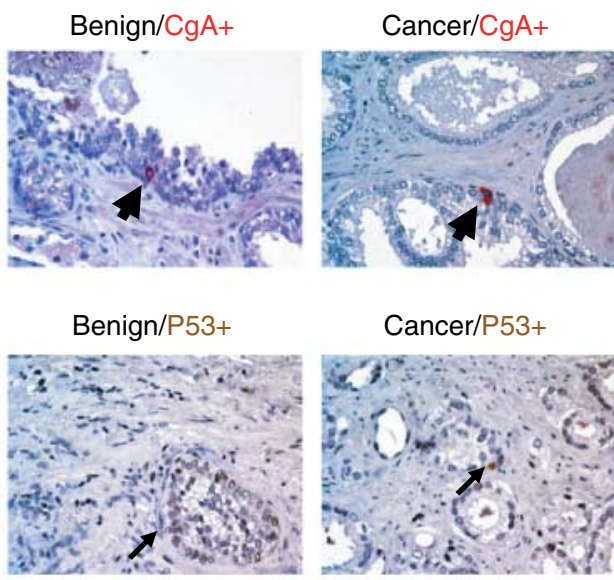

Benign/CgA+/P53+

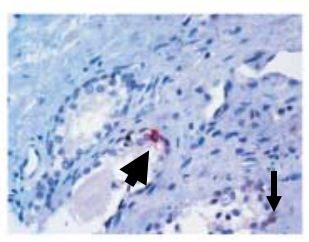

Cancer/CgA+/P53+

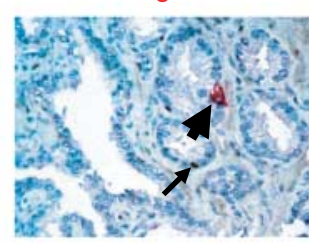

Figure 3 NE cells in benign and adenocarcinoma of prostate are negative for nuclear P53 staining. (A) Adjacent sections of benign prostate were stained with anti-CgA and anti-P53 antibodies respectively. The upper panel shows that NE cells (CgA +, arrows) are negative for nuclear P53. The lower panel shows that the scattered P53 + cells (arrows) were not NE cells (CgA-). (B) Adjacent sections of prostate adenocarcinoma were stained with anti-CgA and anti-P53 antibodies respectively. The upper panel shows that NE cells (CgA +, arrows) are negative for nuclear P53. The lower panel shows that the scattered P53+ cells (arrows) were not NE cells $(\mathrm{CgA}-)$. (C) Sections of benign prostate and prostate adenocarcinoma were doubly stained for CgA (cytoplasmic, red, thick arrow) and P53 (nuclear, brown, thin arrow). The pictures on the left show benign prostate with NE cells (thick arrow, top), P53+ cells (thin arrow, middle), and both (lower). The right panel shows the same in adenocarcinoma. Importantly, cytoplasmic CgA staining and nuclear P53 staining do not occur in the same cells. 
A

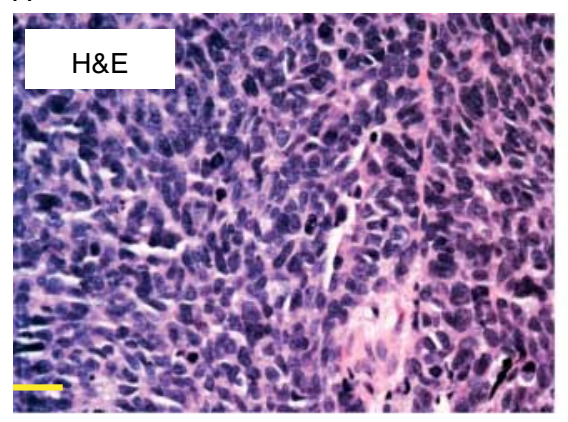

B

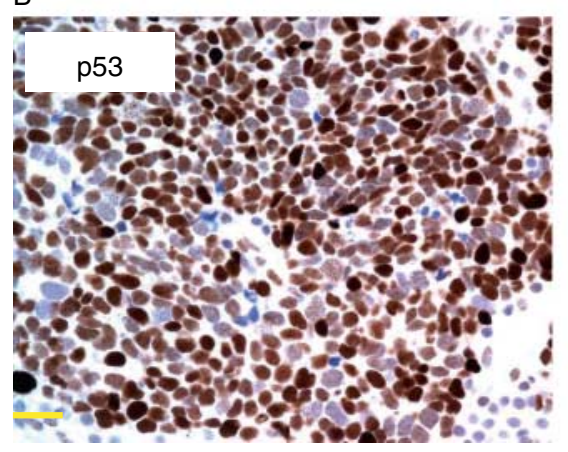

C
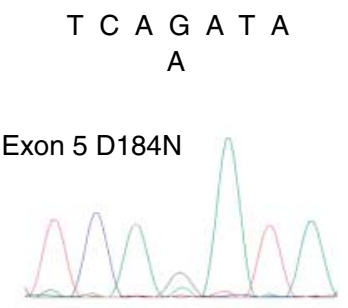

Exon 5 D184N

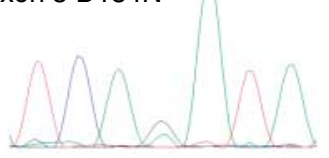

Wild-type p53

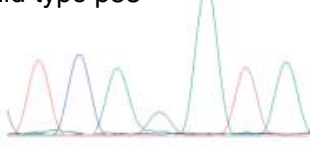

Figure 4 SCNC shows diffuse nuclear P53 staining and contains P53 mutation. (A) H\&E slide of SCNC composed of pure NE tumor cells with characteristic morphological features including scant cytoplasm, fine nuclear chromatin pattern, and nuclear molding. (B) Immunohistochemistry shows diffuse nuclear P53 staining in SCNC. (C) Sequencing data from SCNC. The two examples in the top and middle panels show p53 mutation in exon 5 resulting in replacement of aspartic acid at position 184 by asparagine. The bottom panel shows wild-type P53 sequence in the same region.

a p53 mutation inactivates the IL8-CXCR2-p53 pathway that normally keeps the NE cells in a quiescent state, resulting in hyper-proliferation and aggressive behavior, features that are characteristic of the tumor cells in SCNC.

\section{Discussion}

NE cells are normally present as a minor component in benign prostate and prostatic adenocarcinomas and their functions remain unclear. It has been observed that in cultured LNCaP cells, a cell line with luminal cell features, androgen withdrawal induces differentiation toward a NE phenotype (Burchardt et al. 1999), suggesting that hormonal therapy in PC patients may drive luminal-type cancer cells into NE cancer cells. One interesting hypothesis is that the normally quiescent NE tumor cells may provide growth signals to the adjacent non-NE cancer cells through a paracrine mechanism, contributing to therapeutic failure and the progression to castration-resistant state (Vashchenko \& Abrahamsson 2005, Huang et al. 2007, Yuan et al. 2007, Sun et al. 2009). Occasionally, the recurrent tumor contains pure populations of highly aggressive
NE tumor cells and is classified as SCNC, although SCNC can also be seen in patients without a history of adenocarcinoma. With the advent of novel drugs such as Abiraterone and MDV3100 that show superior efficacy in the inhibition of AR signaling, we expect that the incidence of prostatic SCNC will only increase.

The cell of origin and the molecular basis for prostatic adenocarcinoma remain controversial. These issues are even less clear for prostatic SCNC. Our results are consistent with a model in which the NE cells in benign prostate and prostatic adenocarcinoma are normally quiescent due to the growth inhibitory function of an autocrine mechanism involving the IL8CXCR2-p53 pathway. Mutation of p53 leads to inactivation of the above pathway leading to hyperproliferation of NE cells with the resultant SCNC as shown in Fig. 5. In fact, in the absence of functional P53, activation of CXCR2 by IL8 appears to stimulate cell growth (Fig. 2), suggesting that CXCR2 may elicit both growth inhibitory and growth stimulatory pathways. Normally, the P53-mediated growth-inhibitory pathway may dominate. Once P53 is mutated, CXCR2 activation becomes growth promoting and oncogenic. This hypothesis is supported by previous reports showing 

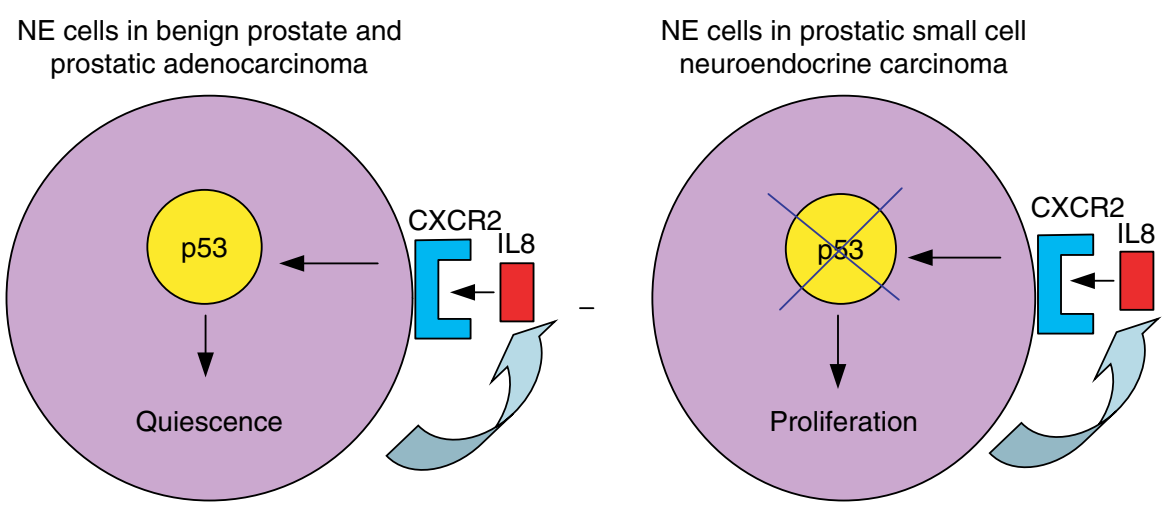

Figure 5 The function of IL8-CXCR2-p53 pathway in controlling the proliferation of NE cells in benign prostate, adenocarcinoma, and SCNC. The model on the left suggests that autocrine activation of CXCR2 by IL8 activates the p53 pathway, which keeps NE cells of benign prostate and prostate adrenocarcinoma in a quiescent state. The model on the right suggests that p53 mutation inactivates the IL8-CXCR2-p53 pathway, leading to rapid proliferation and aggressive biological behavior of NE tumor cells in SCNC.

that a CXCR2-neutralizing antibody can inhibit PC3 cell proliferation (Reiland et al. 1999) and deletion of CXCR2 inhibits TRAMP tumors (Shen et al. 2006).

Therefore, we have identified a potential cellof-origin as well as a molecular target for prostatic SCNS. It is unclear whether the NE cells in both benign prostate and adenocarcinoma can be the cells of origin for SCNS. It is possible that pure SCNC arising de novo may result from $p 53$ mutation in NE cells of benign prostate, although we cannot exclude the possibility that SCNC always arises from NE cells of adenocarcinoma, but in some cases, the rapidly proliferating tumor NE cells have completely overtaken the slow-growing adenocarcinoma, resulting in the histological appearance of a pure SCNC. For those SCNCs that coexist with prostatic adenocarcinoma and those that are recurrent tumors in patients with a history of adenocarcinoma treated with hormonal therapy, the most likely mechanism appears to be p53 mutation in the NE cells of adenocarcinoma. For these patients, a likely scenario is that within the adenocarcinoma, a p53 mutation occurs in NE cell(s) during the course of hormonal therapy leading to the development of SCNC. The rapidly proliferating NE cells of SCNC can coexist with adenocarcinoma initially but eventually become the only component as adenocarcinoma cells usually have a low proliferation rate and this component can be completely overtaken by SCNC. Because of its distinct cell of origin and molecular alteration, SCNC is an entirely different disease from adenocarcinoma. In contrast to adenocarcinoma, prostatic SCNC is a disease of NE differentiation, likely has its cell of origin in NE cells of benign prostate and/or adenocarcinoma, with $p 53$ mutation as the underlying molecular mechanism, and does not respond to hormonal therapy, which has proven to be effective, at least initially, in nearly all patients with prostatic adenocarcinomas.

Our results are consistent with findings in cell line models. We have shown that PC3 cells, a PC cell line originally derived from a patient's bone metastasis, have NE features in that they express NE markers CgA and NSE (Palapattu et al. 2009). Like NE cells in human prostate and prostatic adenocarcinoma, PC 3 cells express IL8 (Ma et al. 2009) and IL8 receptor CXCR2 (Reiland et al. 1999) and are positive for CD44 (Palapattu et al. 2009). Unlike NE cells in benign prostate and adenocarcinoma, PC3 cells are highly proliferative and biologically aggressive, features shared by SCNCs. Consistent with this notion, it has been shown that PC3 cells contain a p53 mutation and restoration of $\mathrm{p} 53$ function inhibits tumor cell growth (Isaacs et al. 1991). Therefore, we have proposed that PC 3 cells may actually represent a cell line of SCNC (Tai et al. 2011).

Our model is supported by animal models of PC. Transgenic mice expressing SV40 early genes including $\mathrm{T}$ antigen (TRAMP) develop aggressive carcinomas with abundant NE tumor cells similar to human prostatic SCNC (Greenberg et al. 1995, Masumori et al. 2001, 2004), as do $p 53^{-/-} R b^{-/-}$double knockout mice (Zhou et al. 2006). In both models, the AR-responsive probasin promoter was used to drive the expression of the transgene or the Cre recombinase. Although AR is not usually expressed in NE cells, it is possible that a low level of AR is present in NE cells at some point of mouse prostate development and is sufficient to activate the probasin promoter. We hypothesize that in these animals, inactivation of $\mathrm{p} 53$ in NE cells of the prostate leads to malignant transformation and rapid proliferation of 
NE cells, resulting in the development of a malignant tumor with abundant NE tumor cells mimicking human prostatic SCNC. Notably, in both models, there are also areas of glandular formation resembling adenocarcinoma, which probably results from inactivation of p53 and/or $\mathrm{Rb}$ in luminal epithelial cells. In most of the TRAMP tumor tissues we have examined, NE tumor cells predominate, likely reflecting the highly proliferative nature of the malignant NE tumor cells.

The findings described here are also consistent with a previous report by De Marzo's group who reported positive nuclear staining for $\mathrm{p} 53$ and a $p 53$ mutation in a case of SCNC intermixed with adenocarcinoma (Hansel et al. 2009). The p53 mutation discovered in their study differs from that identified in our cases, suggesting that a variety of $p 53$ mutations may be found if a large number of SCNCs are sequenced.

It is noteworthy that SV40 T antigen inactivates both p53 and Rb in TRAMP tumors and the Nikitin group has shown that both p53 and $\mathrm{Rb}$ need to be inactivated in order for invasive tumors to develop (Zhou et al. 2006). It is possible that, inactivation of $\mathrm{Rb}$ as well as p53 in NE cells is required for the development of prostatic SCNC in men. However, unlike p53 for which mutations are usually associated with protein accumulation in the nucleus detectable by immunohistochemistry, assaying for the inactivation of $\mathrm{Rb}$ pathway is not straightforward. Therefore, additional studies need to be performed to determine whether the Rb pathway, which appears to be important for prostatic adenocarcinoma (Balk \& Knudsen 2008), is also involved in the pathogenesis of prostatic SCNC in men.

\section{Declaration of interest}

The authors declare that there is no conflict of interest that could be perceived as prejudicing the impartiality of the research reported.

\section{Funding}

J Huang is supported by Department of Defense Prostate Cancer Research Program (PC1001008), UCLA SPORE in prostate cancer (PI: R Reiter), a challenge award from the Prostate Cancer Foundation (PI: O Witte), a creativity award from Prostate Cancer Foundation (PI: M Rettig), and NIH 1R01CA158627 (PI. L Marks).

\section{Author contribution statement}

H Chen, Y Sun, L Cheng, C Wu, J L Yao, S Shen, A O Osunkoya, C Liang, and J Huang designed the study; HChen, Y Sun, and C Wu performed the experiments; Y Sun, CE Magyar, $\mathrm{X} \mathrm{Li}$, and $\mathrm{J}$ Huang analyzed the data; $\mathrm{J}$ Huang wrote the manuscript.

\section{Acknowledgement}

We thank Dr Schraufstatter of La Jolla Institute for Molecular Medicine for providing the $C X C R 2 \mathrm{cDNA}$ and Ms Qi Yang, Ngan Doan, and Ko Kiehle for performing immunohistochemical studies.

\section{References}

Acosta JC, O’Loghlen A, Banito A, Guijarro MV, Augert A, Raguz S, Fumagalli M, Da Costa M, Brown C, Popov N et al. 2008 Chemokine signaling via the CXCR2 receptor reinforces senescence. Cell 133 1006-1018. (doi:10.1016/ j.cell.2008.03.038)

Balk SP \& Knudsen KE 2008 AR, the cell cycle, and prostate cancer. Nuclear Receptor Signaling 6 e001.

van Bokhoven A, Varella-Garcia M, Korch C, Johannes WU, Smith EE, Miller HL, Nordeen SK, Miller GJ \& Lucia MS 2003 Molecular characterization of human prostate carcinoma cell lines. Prostate 57 205-225. (doi:10.1002/ pros.10290)

Bonkhoff H 2001 Neuroendocrine differentiation in human prostate cancer. Morphogenesis, proliferation and androgen receptor status. Annals of Oncology 12 (Suppl 2) S141-S144. (doi:10.1093/annonc/12.suppl_2.S141)

Brown JR, Wieczorek TJ, Shaffer K \& Salgia R 2003 Smallcell cancers, and an unusual reaction to chemotherapy. Case 1. Extrapulmonary small-cell carcinoma arising in the prostate. Journal of Clinical Oncology 21 2437-2438. (doi:10.1200/JCO.2003.081.03)

Burchardt T, Burchardt M, Chen MW, Cao Y, de la Taille A, Shabsigh A, Hayek O, Dorai T \& Buttyan R 1999

Transdifferentiation of prostate cancer cells to a neuroendocrine cell phenotype in vitro and in vivo. Journal of Urology 162 1800-1805. (doi:10.1016/S0022-5347(05)68241-9)

Cooperberg MR, Park S \& Carroll PR 2004 Prostate cancer 2004: insights from national disease registries. Oncology 18 1239-1247 (discussion 1248-1250, 1256-1258).

Erasmus CE, Verhagen WI, Wauters CA \& van Lindert EJ 2002 Brain metastasis from prostate small cell carcinoma: not to be neglected. Canadian Journal of Neurological Sciences 29 375-377.

Greenberg NM, DeMayo F, Finegold MJ, Medina D, Tilley WD, Aspinall JO, Cunha GR, Donjacour AA, Matusik RJ \& Rosen JM 1995 Prostate cancer in a transgenic mouse. PNAS 92 3439-3443. (doi:10.1073/ pnas.92.8.3439)

Grignon DJ 2004 Unusual subtypes of prostate cancer. Modern Pathology 17 316-327. (doi:10.1038/modpathol.3800052)

Hansel DE, Nakayama M, Luo J, Abukhdeir AM, Park BH, Bieberich CJ, Hicks JL, Eisenberger M, Nelson WG, Mostwin JL et al. 2009 Shared TP53 gene mutation in morphologically and phenotypically distinct concurrent primary small cell neuroendocrine carcinoma and adenocarcinoma of the prostate. Prostate 69 603-609. (doi:10.1002/pros.20910)

Huang J, Yao JL, Zhang L, Bourne PA, Quinn AM, di Sant' Agnese PA \& Reeder JE 2005 Differential 
expression of interleukin- 8 and its receptors in the neuroendocrine and non-neuroendocrine compartments of prostate cancer. American Journal of Pathology 166 1807-1815. (doi:10.1016/S0002-9440(10)62490-X)

Huang J, Yao JL, di Sant'agnese PA, Yang Q, Bourne PA \& $\mathrm{Na}$ Y 2006 Immunohistochemical characterization of neuroendocrine cells in prostate cancer. Prostate 66 1399-1406. (doi:10.1002/pros.20434)

Huang J, Wu C, di Sant'Agnese PA, Yao JL, Cheng L \& $\mathrm{Na}$ Y 2007 Function and molecular mechanisms of neuroendocrine cells in prostate cancer. Analytical and Quantitative Cytology and Histology 29 128-138.

Isaacs WB, Carter BS \& Ewing CM 1991 Wild-type p53 suppresses growth of human prostate cancer cells containing mutant p53 alleles. Cancer Research 51 4716-4720.

Kudahetti S, Fisher G, Ambroisine L, Foster C, Reuter V, Eastham J, Moller H, Kattan MW, Cooper CS, Scardino P et al. 2009 p53 immunochemistry is an independent prognostic marker for outcome in conservatively treated prostate cancer. BJU International 104 20-24. (doi:10.1111/j.1464-410X.2009.08407.x)

Ma J, Ren Z, Ma Y, Xu L, Zhao Y, Zheng C, Fang Y, Xue T, Sun B \& Xiao W 2009 Targeted knockdown of EGR-1 inhibits IL-8 production and IL-8-mediated invasion of prostate cancer cells through suppressing EGR-1/ NF-kappaB synergy. Journal of Biological Chemistry 284 34600-34606. (doi:10.1074/jbc.M109.016246)

Masumori N, Thomas TZ, Chaurand P, Case T, Paul M, Kasper S, Caprioli RM, Tsukamoto T, Shappell SB \& Matusik RJ 2001 A probasin-large T antigen transgenic mouse line develops prostate adenocarcinoma and neuroendocrine carcinoma with metastatic potential. Cancer Research 61 2239-2249.

Masumori N, Tsuchiya K, Tu WH, Lee C, Kasper S, Tsukamoto T, Shappell SB \& Matusik RJ 2004 An allograft model of androgen independent prostatic neuroendocrine carcinoma derived from a large probasin promoter-T antigen transgenic mouse line. Journal of Urology 171 439-442. (doi:10.1097/01.ju.0000099826. 63103.94)

Miyoshi Y, Uemura H, Kitami K, Satomi Y, Kubota Y \& Hosaka M 2001 Neuroendocrine differentiated small cell carcinoma presenting as recurrent prostate cancer after androgen deprivation therapy. BJU International $\mathbf{8 8}$ 982-983. (doi:10.1046/j.1464-4096.2001.00936.x)

Palapattu GS, Wu C, Silvers CR, Martin HB, Williams K, Salamone L, Bushnell T, Huang L-S, Yang Q \& Huang J 2009 Selective expression of CD44, a putative prostate cancer stem cell marker, in neuroendocrine tumor cells of human prostate cancer. Prostate 69 787-798. (doi:10.1002/pros.20928)

Reiland J, Furcht LT \& McCarthy JB 1999 CXC-chemokines stimulate invasion and chemotaxis in prostate carcinoma cells through the CXCR2 receptor. Prostate 41 78-88. (doi:10.1002/(SICI)1097-0045(19991001)41:2<78::AID -PROS2 > 3.0.CO;2-P)
Scher HI 2003 Prostate carcinoma: defining therapeutic objectives and improving overall outcomes. Cancer 97 758-771. (doi:10.1002/cncr.11151)

Schlomm T, Iwers L, Kirstein P, Jessen B, Kollermann J, Minner S, Passow-Drolet A, Mirlacher M, Milde-Langosch K, Graefen M et al. 2008 Clinical significance of p53 alterations in surgically treated prostate cancers. Modern Pathology 21 1371-1378. (doi:10.1038/modpathol.2008.104)

Shen H, Schuster R, Lu B, Waltz SE \& Lentsch AB 2006 Critical and opposing roles of the chemokine receptors CXCR2 and CXCR3 in prostate tumor growth. Prostate 66 1721-1728. (doi:10.1002/pros.20476)

Sun Y, Niu J \& Huang J 2009 Neuroendocrine differentiation in prostate cancer. American Journal of Translational Research 1 148-162.

Tai S, Sun Y, Squires JM, Zhang H, Oh WK, Liang CZ \& Huang J 2011 PC3 is a cell line characteristic of prostatic small cell carcinoma. Prostate 71 1668-1679. (doi:10.1002/pros.21383)

Tanaka M, Suzuki Y, Takaoka K, Suzuki N, Murakami S, Matsuzaki O \& Shimazaki J 2001 Progression of prostate cancer to neuroendocrine cell tumor. International Journal of Urology 8 431-436 (discussion 437). (doi:10.1046/j.1442-2042.2001.00347.x)

Vashchenko N \& Abrahamsson PA 2005 Neuroendocrine differentiation in prostate cancer: implications for new treatment modalities. European Urology 47 147-155. (doi:10.1016/j.eururo.2004.09.007)

Wang W \& Epstein JI 2008 Small cell carcinoma of the prostate: a morphologic and immunohistochemical study of 95 cases. American Journal of Surgical Pathology 32 65-71. (doi:10.1097/PAS.0b013e318058a96b)

Wu C, Zhang L, Bourne PA, Reeder JE, di Sant'agnese PA, Yao JL, Na Y \& Huang J 2006 Protein tyrosine phosphatase PTP1B is involved in neuroendocrine differentiation of prostate cancer. Prostate 66 1125-1135. (doi:10.1002/pros.20412)

Yao JL, Madeb R, Bourne P, Lei J, Yang X, Tickoo S, Liu Z, Tan D, Cheng L, Hatem F et al. 2006 Small cell carcinoma of the prostate: an immunohistochemical study. American Journal of Surgical Pathology 30 705-712. (doi:10.1097/00000478-200606000-00005)

Yuan TC, Veeramani S \& Lin MF 2007 Neuroendocrine-like prostate cancer cells: neuroendocrine transdifferentiation of prostate adenocarcinoma cells. Endocrine-Related Cancer 14 531-547. (doi:10.1677/ERC-07-0061)

Zhou Z, Flesken-Nikitin A, Corney DC, Wang W, Goodrich DW, Roy-Burman P \& Nikitin AY 2006 Synergy of p53 and $\mathrm{Rb}$ deficiency in a conditional mouse model for metastatic prostate cancer. Cancer Research 66 7889-7898. (doi:10.1158/0008-5472.CAN-06-0486)

Received in final form 17 February 2012 Accepted 29 February 2012 Made available online as an Accepted Preprint 2 March 2012 